\title{
CASS Family TPS Support Toolset
}

\author{
Gilberto García, Robert Loe, Brad Bravo, José Cifredo \\ Fleet Readiness Center Southeast (FRCSE) \\ 6206 Aviation Avenue, Building 6206, Room J-2 \\ Jacksonville, FL 32221
}

Phone: (904)-317-1682, Email: \{gilberto.garcia1, robert.loe, brad.bravo, jose.cifredo\}@navy.mil

\begin{abstract}
Migration, maintenance and regression of more than three hundred (300) Test Program Set (TPS) to support all existing (i.e., Consolidated Automated Support System (CASS) and Reconfigurable Transportable Consolidated Automated Support System (RTCASS)) and future (e.g., electronic Consolidated Automated Support System (eCASS)) CASS Family Automated Test Equipment (ATE) members can be an overwhelming task that requires compilation and build of source, translation, data transfer and data validation.
\end{abstract}

CASS family automation tools can help TPS developers create and execute from Personal Computer (PC) environment CASS Virtual Address eXtension (VAX) Digital Command Line (DCL) scripting files without physically being on an ATE station; make Magneto-Optical (MO) disks (without user intervention) from VAX backup save set files; make automated Version Description Document (VDD) reports at Operational Test Program Set (OTPS) level; identify and fix corrupted Master Test Program Set Index (MTPSI) files; identify corrupted CASS Abbreviated Test Language for All Systems (ATLAS) files; identify missing CASS files (ATLAS source ATLAS lookup, graphics, backup save set, Digital Test Language, Digital Automated Test Program Generation (DATPG), shutdown, data, subassembly and Functional Extension Program); validate and create Automated Test System (ATS) Source Data Repository (ASDR) directory structure; create file list inventories for Configuration Management (CM) and Quality Assurance (QA); have a visual representation of the MTPSI card; and provide support for the Next Generation Operations Management Software (NxOMS) communication.

This paper will demonstrate improvements from our previous suite of automation tools, before and after figures showing tools benefits, and faced hardware challenges.

Keywords - OTPS Migration; ATLAS; CASS VAX/VMS DCL; Automation tools; NxOMS; RTCASS ToolBox; ASDR; MTPSI.

\section{INTRODUCTION}

CASS is the Navy's standard Automatic Test System (ATS) and has been designated by the Naval Air Systems Command for the support of avionics/electronics systems at Navy Intermediate Level maintenance and Fleet Readiness Center (FRC) sites both ashore and afloat. The newest member of CASS family RTCASS, is a portable, readily deployable version of CASS that is being produced to support U.S. Marine Corps and U.S. Special Operations Command
(US SOCOM) V-22 as well as F/A-18, EA-6B, and AV-8B aircraft[1]. The future member of CASS family, eCASS, will replace the five mainstream CASS configurations (Hybrid, Radio Frequency (RF), Communications/Navigation/Interrogation (CNI), High Power (HP) and Electro-Optic (EO)) [2].

FRCSE Jacksonville, FL was tasked by Naval Air Systems Command (PMA260) to assist in the TPS Migration process from CASS to RTCASS that includes standardization of CASS ATLAS source files and graphics; translation of CASS ATLAS, CASS DATPG, Digital Test Language and graphics (as known as convert to "Green"); regenerate source from CASS Digital Test Language and DATPG runtime files; compile CASS source files; verify log files for errors at compilation; transfer TPS source files from MO or "Blue Disk" to the PC environment considering different network connectivity (Navy Marine Corps Intranet (NMCI) and Research, Development, Test and Evaluation (RDT\&E)) at different locations; update CASS source and VDD in ASDR; and release new changes to "Blue Disk" and "Green Disk" (Fig. 1).

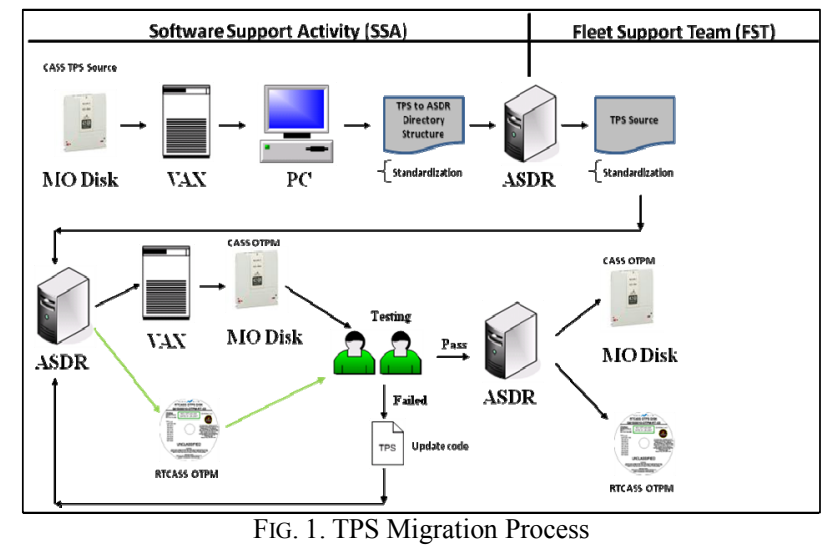

To accomplish the TPS migration task, FRCSE Jacksonville, FL created various software tools to automate repetitive processes while at the same time minimize probability of errors, and deliver TPS at quick turnaround time. This paper updates and expands the automation tools concept introduced in a previous paper [3]; describes automation process and tools for TPSs migration, maintenance and regression. 


\section{AUTOMATED PROCESS}

Automated processes significantly minimize the process by scripting VAX DCL command files, automating creation of VDD reports, automating file transfer procedures from/to PC to/from VAX among other. In contrast with the manual process, an automated process is transparent to the user. Therefore, the user is not required to have VAX knowledge. The process step is shown in Table 1.

TABLE I. Manual And Automated Process

\begin{tabular}{|c|c|c|}
\hline \multirow{2}{*}{ Step } & \multicolumn{2}{|c|}{ Process } \\
\hline & Manual & Automated \\
\hline Download TPS from ASDR to PC & $\sqrt{ }$ & \\
\hline $\begin{array}{l}\text { Change TPS source files on PC. Edit } \\
\text { TPS source code with new } \\
\text { modifications. Replace messages and } \\
\text { prases related to CASS ATLAS and } \\
\text { graphics. }\end{array}$ & & $\sqrt{ }$ \\
\hline $\begin{array}{l}\text { Translate TPS to Test Program Markup } \\
\text { Language (TPML) using RTCASS } \\
\text { Toolbox. }\end{array}$ & & $\sqrt{ }$ \\
\hline $\begin{array}{l}\text { After translation, check for errors and } \\
\text { warnings. }\end{array}$ & $\sqrt{ }$ & \\
\hline $\begin{array}{l}\text { Convert legacy MTPSI file to TPSI } \\
\text { format. }\end{array}$ & & $\sqrt{ }$ \\
\hline $\begin{array}{l}\text { Create directory in VAX. Create in } \\
\text { TPSWSDIR TPS directory and L2Text } \\
\text { Directory job and segment. }\end{array}$ & & $\sqrt{ }$ \\
\hline Depending on site, use SFTP or FTP ${ }^{*}$. & & $\sqrt{ }$ \\
\hline $\begin{array}{l}\text { Identify binary and American Standard } \\
\text { Code for Information Interchange } \\
\text { (ASCII) file type prior transfer }{ }^{*} \text {. }\end{array}$ & & $\sqrt{ }$ \\
\hline $\begin{array}{l}\text { Send TPS to VAX. Use Terminal } \\
\text { emulator software to transfer individual } \\
\text { CASS source files. Files are sent } \\
\text { directly (in the case of RDT\&E) to the } \\
\text { VAX or through CODERS. }\end{array}$ & & $\sqrt{ }$ \\
\hline $\begin{array}{l}\text { Compile TPS. Compile CASS ATLAS, } \\
\text { DATPG and Digital Test Language. }\end{array}$ & & $\sqrt{ }$ \\
\hline Check log files for compilation errors. & $\sqrt{ }$ & \\
\hline Make Blue Disk. & & $\sqrt{ }$ \\
\hline $\begin{array}{l}\text { Create an inventory list of files } \\
\text { contained in } \mathrm{MO} \text { for } \mathrm{CM} \text { and QA } \\
\text { review. }\end{array}$ & & $\sqrt{ }$ \\
\hline $\begin{array}{l}\text { Compress VAX backup save set(s) and } \\
\text { MTPSI file(s). }\end{array}$ & & $\sqrt{ }$ \\
\hline Transfer files back to PC. & & $\sqrt{ }$ \\
\hline $\begin{array}{l}\text { Generate a VDD between baseline and } \\
\text { new file set }\end{array}$ & & $\sqrt{ }$ \\
\hline Upload OTPS/TPS and VDD to ASDR. & $\sqrt{ }$ & \\
\hline
\end{tabular}

The design of the tools considered different operating systems, file transfer protocols, and ATE experience. Depending on the stage in the process, a set of tools was created to maximize productivity while integrating third-party applications. Figure 2 shows the Automated Maintenance of OTPS/TPS Migration Process cycle after the SSA upload the OTPS/TPS into ASDR.

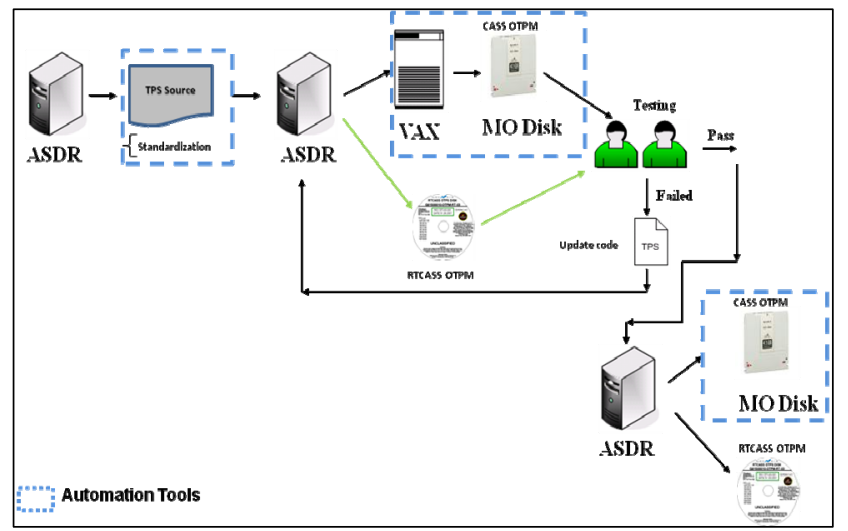

FIG. 2. Automated Maintenance of OTPS/TPS Migration Process

\section{TPS MIGRATION TOOLS}

The migration tools, intended to be used by the Fleet Support Team (FST), are used in a one-time process that involves TPS source code standardization, source file cleanup and conversion.

\section{A. TPS Standardization}

\section{1) Replacer}

Replacer is a PC command line application that replaces legacy CASS ATLAS instructions, terms, and phrases with an equivalent one suitable for common ATE.

\section{2) CASSGRAF}

CASSGRAF is a TPS development tool that performs a wide variety of tasks:

a) Convert various PC graphics formats (PCX, BMP, GIF, JPG and DXF) to the monochrome CASS GRA format.

b) Perform basic image editing functions like cropping, scaling, rotating and inverting.

c) Generate and review automated technical information (ATI) files in CASS format.

d) Convert CASS ATI files to HyperText Markup Language (HTML) format and check for errors.

e) Convert TPS source code (ATLAS and L200"TM) to HTML with links for lookup entries, procedure calls, GOTOs, and REQUIRE labels. Reference to GRA files may optionally be replaced with links to GIF files.

f) Generate test oriented wire list (TOWL) data from TPS source files. Create ATLAS callout messages for Interface Device (ID) self test programs and list undetected wire segments.

g) Display the graphic TOWL (gTOWL) that offers automatic high-lighting of TOWL wire segments.

h) Convert OrCAD Electronic Design Interchange Format (EDIF) output files into gTOWL drawings automatically, and allow the user to manually enter gTOWL wire information.

i) Offers a text based command interface to perform various functions and supports scripting of these commands. In addition, certain commands may be 
executed in a non Graphical User Interface (GUI) mode from the Windows ${ }^{\circledR}$ command prompt.

j) Allows the user to view and extract the contents of Virtual Memory System (VMS) backup save set files stored on the PC.

\section{B. Source file cleanup}

\section{1) TPSCleaner}

TPSCleaner, as the name implies, deletes additional files that are not CASS source. The final result expects CASS graphics (.gra), ATLAS files (.as), lookup files (.lu) and MTPSI files (.dat/.tpsi). This tool also clears L200 ${ }^{\mathrm{TM}}$ and LASAR $^{\mathrm{TM}}$ directories, having only stimulus and pattern files (.sym, .led and .ped) and pinmap (.pmp).

\section{Conversion}

\section{1) VisualMTPSI}

VisualMTPSI converts legacy CASS MTPSI DAT file to the TPSI format. As part of this process, the TPSI files are validated with an XML schema to catch any errors present in the original DAT file.

\section{TPS MAINTENANCE TOOLS}

The maintenance tools are intended to be used by the FST during the sustainment part of the TPS life cycle. TPS developer could use these tools in order to conform to ASDR rules.

\section{1) Tools}

Tools is a PC command line utility tool with the following parameters: MAKETPSBUILD, MAKEGETCDS, MAKEOTPSLST, MAKERTCASSZIP, LOSTGRA, CHECKTPSBUILD, CONVERTDAT, SENDTPS, FETCHTPS, SENDFILES, FETCHFILES and CODEHTML (Fig. 3).

TOOLS - Version $1.33-02 / 01 / 2011-$ Robert Loe
Usage notes:
TOOLS MAKETPSBUILD [tpsdirectory]
TOOLS MAKETPSBUILD [otpsdirectory [MAKECDS]]
TOOLS MAKEGETCDS [otpsdirectory]
TOOLS MAKEOTPSLST [otpsdirectory]
TOOLS MAKERTCASSZIP [otpsdirectory]
TOOLS LOSTGRA [otpsdirectory]
TOOLS LOSTGRA [topleveldirectory TOP]
TOOLS CHECKTPSBUILD [tpsdirectory]
TOOLS CHECKTPSBILD [otpsdirectory MULTIPLE]
TOOLS CONUERTDAT [tpsdirectory]
TOOLS CONUERTDAT [otpsdirectory MULTIPLE]
TOOLS SENDTPS [tpsdirectory]
TOOLS FETCHTPS [tpsdirectory]
TOOLS SENDFILES [anyleveldirectory]
TOOLS FETCHFILES [anyleveldirectory]
TOOLS CODEHTML [otpsdirectory]
TOOLS CODEHTML [tpSdirectory]

FIG. 3. Tools command line parameters. a) MAKETPSBUILD: In order to compile ATLAS and L200 ${ }^{\mathrm{TM}}$ files, MAKETPSBUILD creates VAX scripts (BUILDATLAS.COM and BUILDL200.COM) with build instructions from TPS source files. This argument also checks for errors and checks for missing CASS graphic files.

b) MAKEGETCDS: Creates a VAX script to get CASS L200 ${ }^{\mathrm{TM}}$ circuit database file if required.

c) MAKEOTPSLST: Creates an OTPS XML file with TPS, MTPSI, errors and warnings. Also set compilation requirements for FEP_ALPHA, FEP_VAX, L200 ${ }^{\mathrm{TM}}$ and LASAR ${ }^{\mathrm{TM}}$. Typical warnings include CASS graphics that might not be required in the ATLAS source, but included in the source files (Fig. 4).

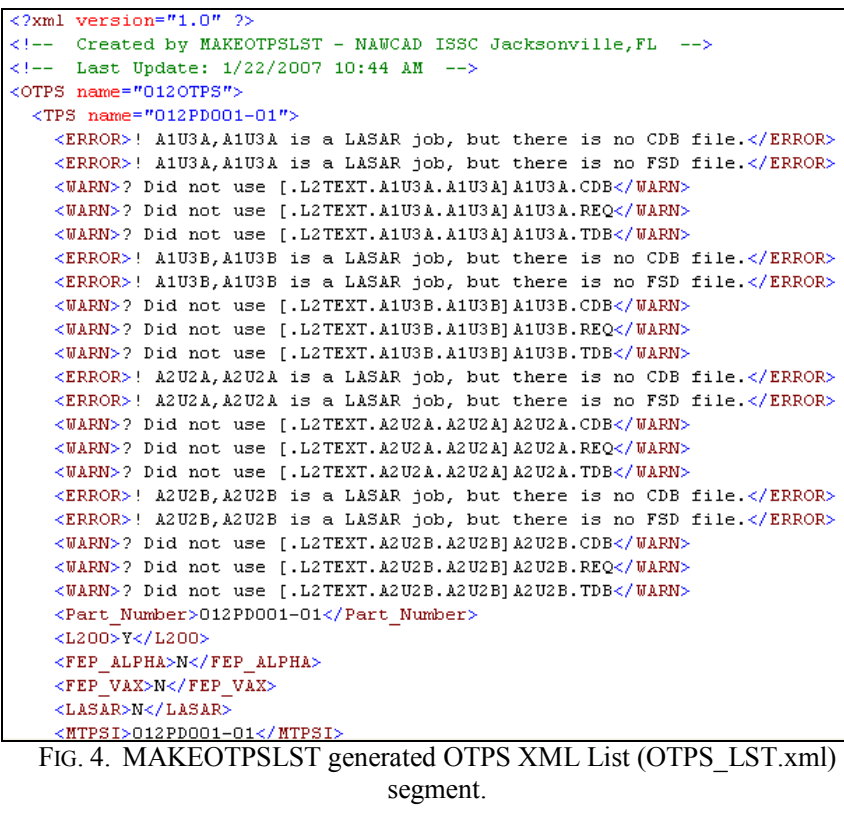

FIG. 4. MAKEOTPSLST generated OTPS XML List (OTPS LST.xml) segment.

d) MAKERTCASSZIP: Creates a DOS batch file to zip and unzip RTCASS \$Convert and \$DTI folders. Also checks if zip and unzip were previously created and validated with TPML files.

e) LOSTGRA: Checks from OTPS level for missing CASS graphic files by parsing the ATLAS code.

f) CHECKTPSBUILD: - Checks for errors at TPS level and generates a report.

g) CONVERTDAT: Converts MTPSI files to TPSI XML format and checks for errors. CONVERTDAT checks in the ATLAS code for UUT requirements, (such as ancillary equipment, part number, support equipment, run-time classification, etc). Before TPSI is created, a pre-validation procedure is done to ensure that instruments are validated.

h) SENDTPS and SENDFILES: Sends TPS files from $\mathrm{PC}$ to VAX through SSH tunnel.

i) FETCHTPS and FETCHFILES: Gets TPS files from $\mathrm{PC}$ to VAX through SSH tunnel.

j) CODEHTML: Generates HTML version of ATLAS source code with links of procedure name, procedure 
calls, test number, UUT requirement, CASS Digital Test Language and DATPG files and statements, used to populate Operational Test Program Instruction (OTPI).

\section{2) FTP2Coders}

FTP2Coders facilitates file transfer from PC to VAX server by having a pre-defined directory structure in both ends and easy configuration of server protocol and file extension type. FTP2Coders uses Secure File Transfer Protocol (SFTP) and File Transfer Protocol (FTP) to connect through CASS OMS Developer Engineering Resource Suite (CODERS) server the file transfer.

\section{3) LogicalsCreator}

LogicalsCreator, a Graphic User Interface (GUI) software PC application, creates the required VAX DCL build scripting files based on compilation options and FEP availability. If used in conjunction with FTP2Coders, it sends the VAX Build scripts necessary to execute in VAX/ALPHA TPS compilation (Fig. 5).

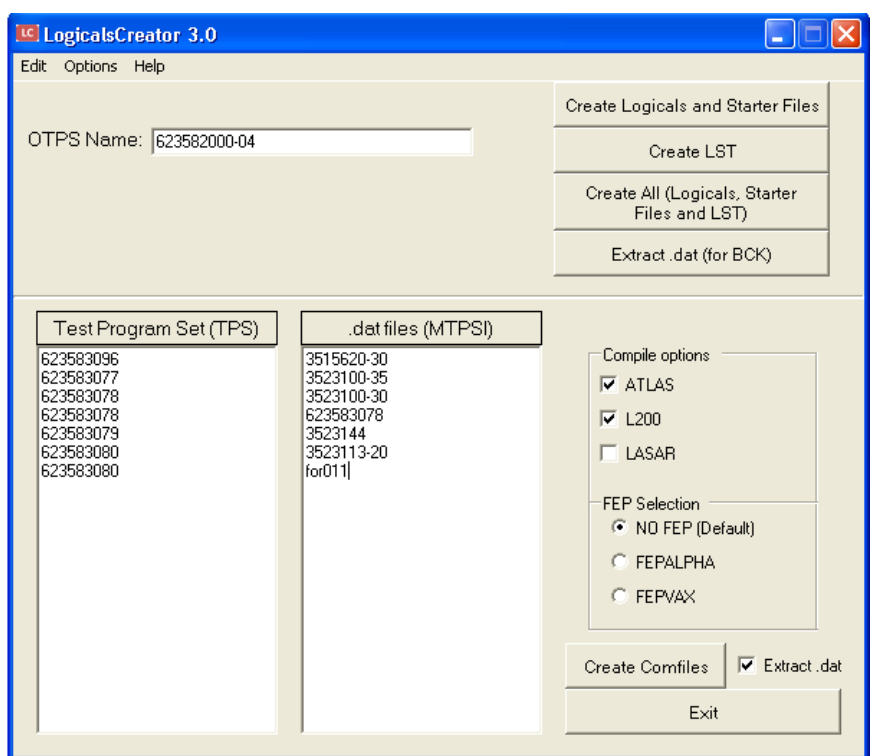

FIG. 5. LogicalsCreator GU

\section{4) Make TPS Extract dats}

Make TPS Extract dats sole function is to fix corrupted MTPSI. This application, executed from DOS command line, takes a list of OTPS, TPS and MTPSI DAT files and then outputs a VAX Script from TPS VAX backup save set files.

\section{5) VAX DCL Scripting}

To reduce the complexity of all VAX operations and to monitor every step in the process, VAX DCL was divided in two processes; VAX Common and Build scripts.

VAX Common and Build scripting files are a set of VAX DCL files organized in two directories (OTPS part number and COMFILES) with the function to compile CASS ATLAS, DATPG and Digital Test Language; build optical disk (Blue Disk) and list VAX backup save set files. VAX Common scripts are pre-defined VAX DCL files with instructions necessary to compile and build a TPS. VAX Build scripts are dynamically generated by Tools and CASSTools (explained next) based on OTPS XML List (OTPS PART NUMBER LST.xml). Both VAX DCL scripts could execute full (Fig. 6), full backup save set only (Fig. 7) or partial process (Fig. 8)

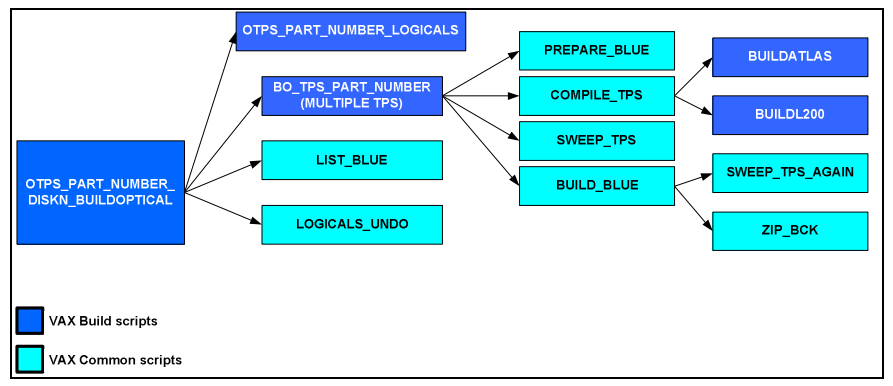

FIG. 6. VAX Build and Common scripts Architecture (Full Process)
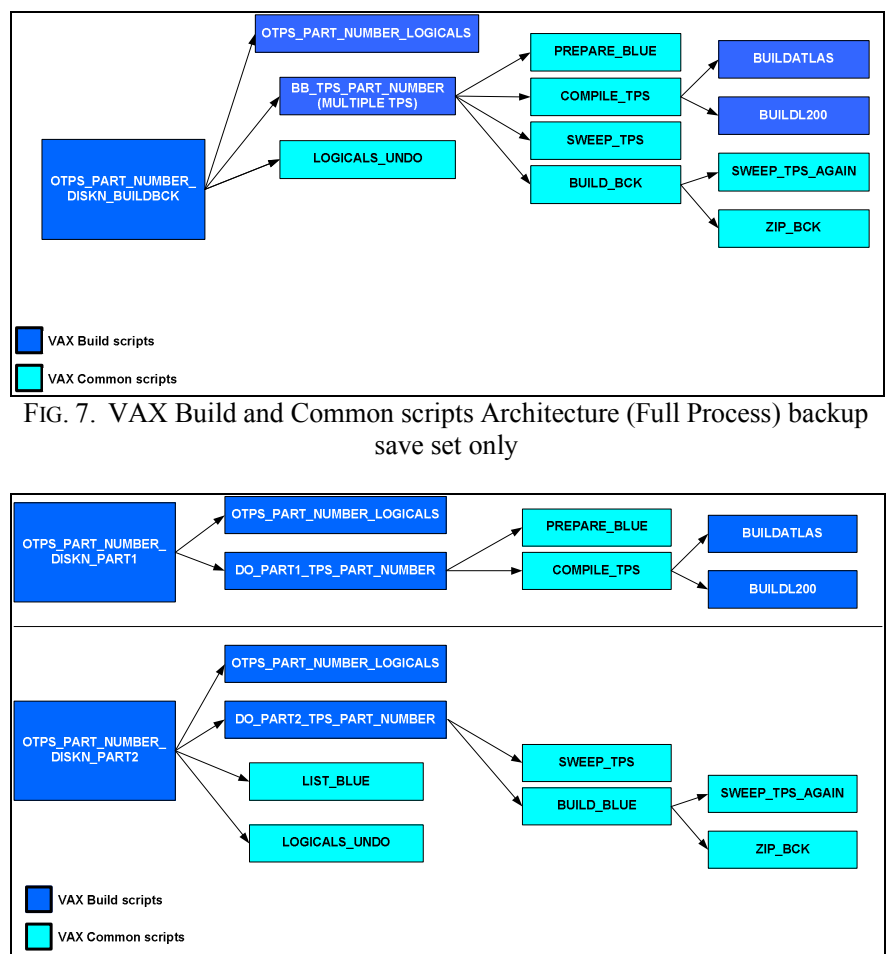

FIG. 8. VAX Build and Common scripts Architecture (Partial Process)

6) CASSTools

CASSTools is the integration of Tools command line, FTP2Coders, LogicalsCreator, MAKE_TPS_EXTRACT_DATS, and VAX DCL Scripting (Fig. 9). First, the application checks if the OTPS directory structure is valid with ASDR directory and file structure. Once the user browses for an OTPS and creates VAX DCL scripts, the rest of the process is transparent, in other words, no manual interactions are required to complete the process. 


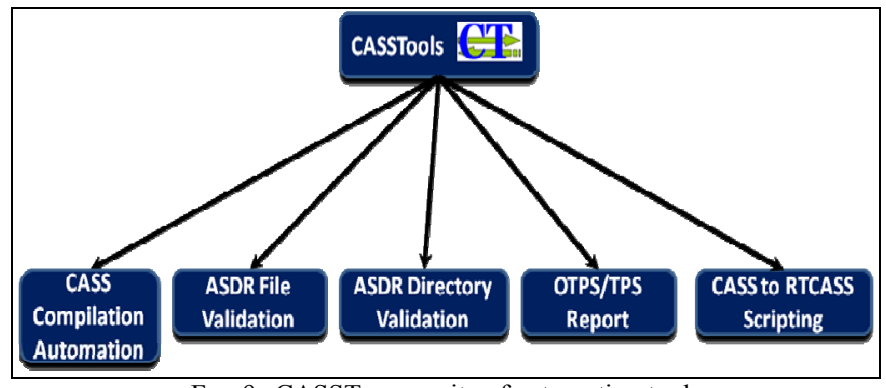

FIG. 9. CASSTOOLS suite of automation tools

Current CASSTools Process includes: Create VAX DCL Files, Send OTPS to VAX, Execute VAX DCL from PC, Get Files from VAX, Make Disk from bck, and Make VDD (Fig. $10)$.

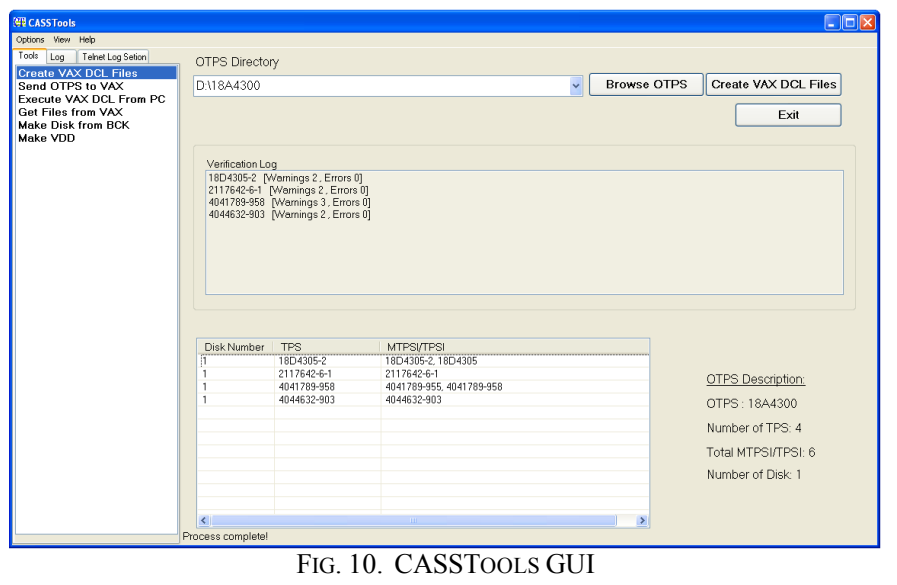

a) Create VAX DCL Files: Creates VAX DCL command files or VAX Build scripts based on OTPS XML List. These generated files allow the user to compile CASS Files and build a Blue Disk.

b) Send OTPS to VAX: Creates a pre-defined transfer directory structure in local PC platform. Then copies VAX Build and Common scripts to the transfer directory. After files are copied, the application checks for BUILDATLAS and BUILDL200 in every TPS and compress \$Convert and \$DTI directory on the TPS. TPS is then copied and compressed in local transfer directory for simple file transfer reasons. Once all files are in local transfer directory, CASSTools verifies for CODERS user Public Key Infrastructure (PKI) certificate and creates one for new users. SSH connection is then established, OTPS directory is created in VAX and files are sent to the VAX. At this point, VAX DCL scripts are ready to be executed.

c) Execute VAX DCL from PC: This function executes VAX DCL scripts from PC by using Cherry Point Telnet Client tool or WRQ Reflections, depending on user selection.

d) Get Files from VAX: Receives TPS VAX backup save set, $\log$ files and list files from VAX. CASSTools connects to CODERS, gets the files and saves them in an expected ASDR directory structure. This process should be used after Blue Disk is created. e) Make Disk from BCK: Sends to VAX backup save set file from the PC to the VAX and creates a MO disk from that file.

f) Make VDD: Compares baseline OTPS file set with the new file set and creates a detailed VDD. The VDD is based on a predefined Microsoft Excel ${ }^{\circledR}$ template which is populated with file changes.

g) CASS to RTCASS: Creates CASS to RTCASS translation scripting and launches RTCASS Toolbox with the generated scripts.

h) NxOMS Message Communicator: Based on NxOMS Software Development Kit (SDK), this application is intended to be used to send files from FRC facilities (Site node) to ATE station(s) (Station node).

\section{7) VisualMTPSI}

VisualMTPSI, in the sustainment life cycle, allows the user to enter MTPSI data into an intuitive graphical interface that looks like a traditional hard-copy MTPSI-card. Context sensitive information is available with a right-click which allows the users to select Operational Test Program Hardware $(\mathrm{OTPH})$ type, ancillary items and station type.

Hard copy output is available to a Windows printer, BMP image file, or Portable Document Format (PDF) file. VisualMTPSI creates PDF files using Adobe ${ }^{\circledR}$ Acrobat ${ }^{\circledR}$ (if available) or by generating a PostScript ${ }^{\circledR}$ file that is converted to PDF by Ghostscript ${ }^{\circledR}$.

On RTCASS, the RESO button executes VisualMTPSI in a special view only mode to display real-time MTPSI data to the operator.

\section{REGRESSION TOOLS}

The regression tools are intended to be used by the Software Support Activity (SSA) to analyze the impact of ATE System Software changes on TPS.

\section{1) TPML Tool}

TPML Tool is used to parse translated ATLAS files (TPML), generated by RTCASS Toolbox, and populate in a table format the resource, action and capability from selected TPS. Once the data is populated the user can sort, select for item of interest, and point into a text editor the selected ATLAS line source code. TPML Tool also exports the data into Microsoft Excel ${ }^{\circledR}$ spreadsheet for easy review; creates a custom XML schema (also known as twd file) for easy parsing of resource usage by test; and generates a Microsoft Excel ${ }^{\circledR}$ spreadsheet with a worksheet of every resource to correlate them with TPS usage for later analysis (Fig. 11). 


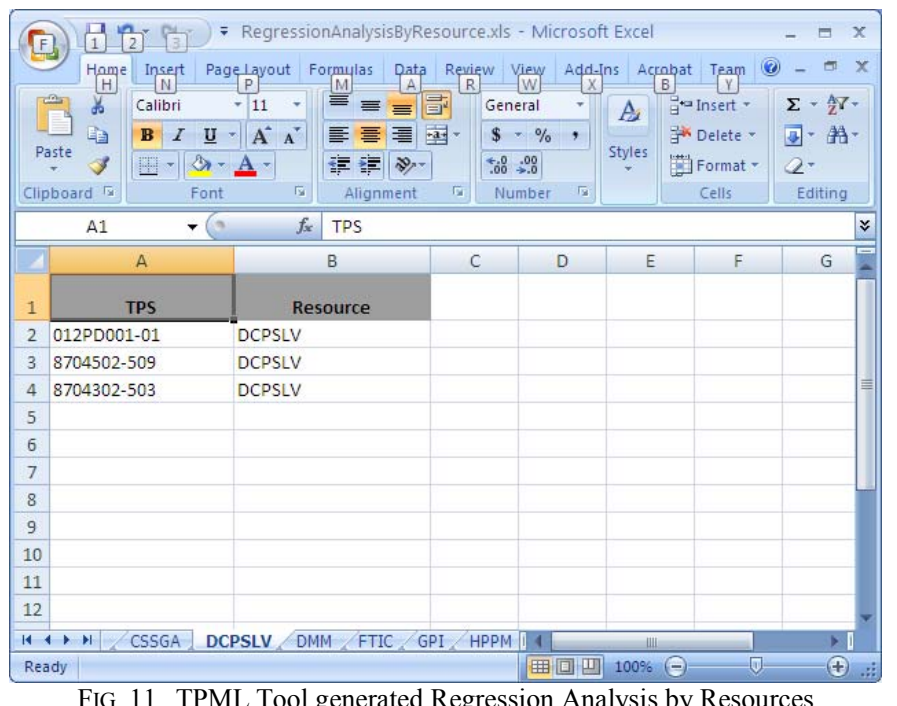

FIG. 11. TPML Tool generated Regression Analysis by Resources

\section{2) TRID Viewer}

The Test and Resource Information Database (TRID) Viewer offers the same functionality as of TPML Tool in addition to resource, capability, action and TPS queries (Fig. 12). Also, TRID Viewer generates a report on Microsoft Excel ${ }^{\circledR}$ for easy review.

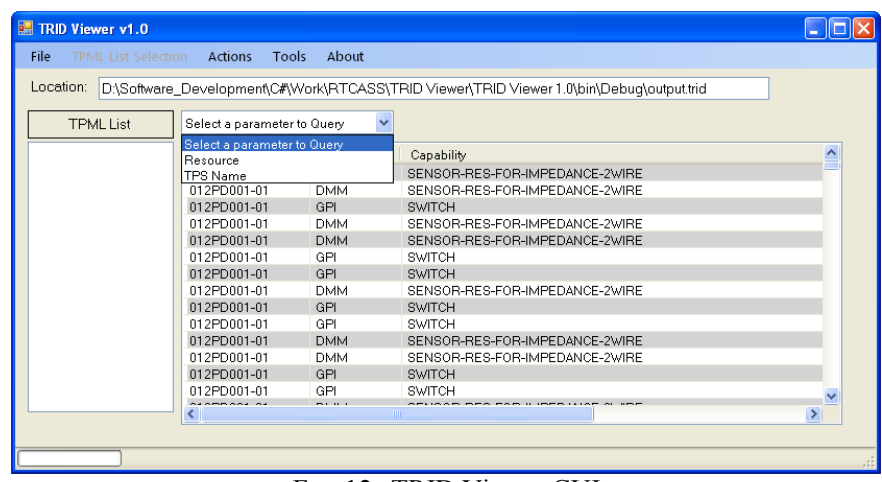

FIG. 12. TRID Viewer GUI

\section{CONCLUSIONS AND RECOMMENDATIONS}

In conclusion, the CASS Family TPS Support toolset can be very useful and cost effective for TPS developers, FSTs and SSAs. Experience has shown that repetitive processes with large amount of TPSs can be very time consuming, less reliable and costly due to the interaction involved in the processes.

In a real scenario, it was noticed that the significant tools contribution came to light after migrating an OTPS that consisted of seventeen (17) TPSs with a mix of analog and digital testing. With the automation tools the overall migration process (Full VAX DCL Process) was completed in about fifty percent $(50 \%)$ of the previous time compared manual process. In another case, a single TPS with just analog testing saved around nineteen percent (19\%) of time compared with manual process (Fig.13).

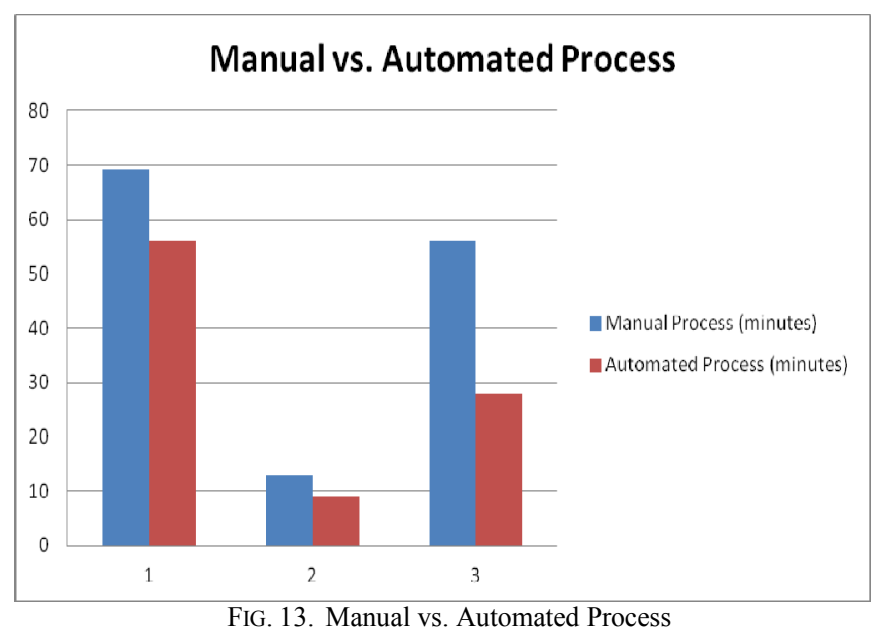

FIG. 13. Manual vs. Automated Process

The real time saver was the automation of user interaction and decisions made during the TPS compilation. There is no automation on error checking after compilation. Depending on user confidence or knowledge with the OTPS/TPS, Full VAX DCL Process is the best suited option.

New and future TPS developers/engineers hired with no experience on VAX VMS, VAX commands, CASS ATLAS, CASS DATPG, Digital Test Language and ASDR could find the tools very attractive since the learning curve required to get familiar with the process. Instead, they can concentrate on developing better TPSs and deploying it at a quick turnaround time.

\section{ACKNOWLEDGEMENT}

The authors would like to thank Timothy Davis, Leo Errico, Jason Bell and Melissa Kelly from FRCSE Jacksonville, FL for contributions on our tools set. Also Robert D. Chase, Stuart W. Rivenbark, Hale Croom and Christopher Sparr from Naval Aviation Depot, Cherry Point, $\mathrm{NC}$ for contributions and ideas offered during early development of CASSTools and RDT\&E telnet client software. John $T$. Olson for providing VAX DCL L2COMGEN and L2SYM for regenerating new L200 source files. Jeff Calalay from Naval Air Station North Island, CA for providing valuable TPS migration data.

Additional thanks to Tony Conard, David Rolke and William Heyn for envisioning and embracing from the beginning our automation tools concept.

\section{REFERENCES}

[1] (2008) The DoD Executive Directorate website. [Online]. Available: http://www.acq.osd.mil/ats.

[2] (2011) Naval Air Systems Command website. [Online]. Available: http://www.navair.navy.mil/index.cfm?fuseaction=home.display\&key= 576C1728-0C54-472F-84B3-CA9D40BBF46D 
[3] García, G., Bravo, B., Cifredo, J, "Automation Tools for CASS and RTCASS", Proc. AUTOTESTCON, Salt Lake City, UT, 2008

Teradyne, TPS Converter Studio $^{\text {TM }}$, M9-Series ${ }^{\text {TM }}$, L-Series ${ }^{\text {TM }}$, $\mathrm{L} 200^{\mathrm{TM}}$ and $\mathrm{LASAR}^{\mathrm{TM}}$ are a trademark of Teradyne, Inc. VMS, AlphaServer and VAX are trademarks of Hewlett Packard. DOS used to represent MS-DOS ${ }^{\mathrm{TM}}$, Windows ${ }^{\circledR}$, Microsoft $\AA$, and Microsoft Excel $\AA$ are trademarks of Microsoft Corporation in the United States and other countries. Adobe $\AA$, Acrobat $\left(\AA\right.$ and PostScript $\left({ }^{\circledR}\right.$ are either registered trademarks or trademarks of Adobe Systems Incorporated in the United States and/or other countries. Ghostscript ${ }^{\circledR}$ is a trademark of Artifex Software, Inc. WRQ and Reflection are registered trademarks of Attachmate Corporation. All other trademarks, trade names, or company names referenced herein are used for identification only and are property of their respective owners. 